$5-1-2021$

\title{
Listen Up! How can social and behavior change programming improve reproductive health and family planning behaviors of out- of-school youth in the Philippines?
}

Breakthrough RESEARCH

Follow this and additional works at: https://knowledgecommons.popcouncil.org/departments_sbsr-pgy How does access to this work benefit you? Let us know!

\section{Recommended Citation}

Breakthrough RESEARCH. 2021. "Listen Up! How can social and behavior change programming improve reproductive health and family planning behaviors of out-of-school youth in the Philippines?" Results Brief. Washington, DC: Population Council. 


\section{How can social and behavior change programming improve reproductive health and family planning behaviors of out-of-school youth in the Philippines?}

The Breakthrough RESEARCH project, in collaboration with De La Salle University's Social Development Research Center in Manila, conducted a targeted study to better understand which social and behavior change programming approaches are most effective for encouraging out-of-school youth (OSY) ages 15 to 19 to adopt priority reproductive health $(\mathrm{RH})$ and family planning (FP) behaviors. The study was conducted among OSY in four urban regions of the Philippines: Manila, Quezon City, Cebu City, and Davao City.

\section{FOUR PRIORITY BEHAVIORS}

Delay of sexual debut

Care seeking in first tri-

mester of pregnant youth

Use of modern contracep-

tive methods to prevent pregnancy
Birth spacing of $3-5$ years
to prevent short birth intervals

\section{Do existing youth programs in the Philippines adequately address the RH and FP needs of OSY?}

- Only 1 of 15 programs focusing on young people in the Philippines covered in this study worked explicitly at the intersection of OSY and adolescent and youth reproductive health (AYRH). Most youth programs focused on life skills or basic education.

- Training and peer education programs dominate the programming landscape.

- Use of innovative information technology and social media in programming approaches is limited.

- The impact of existing programming remains largely unknown, as rigorous program evaluations that measure impact are rare.

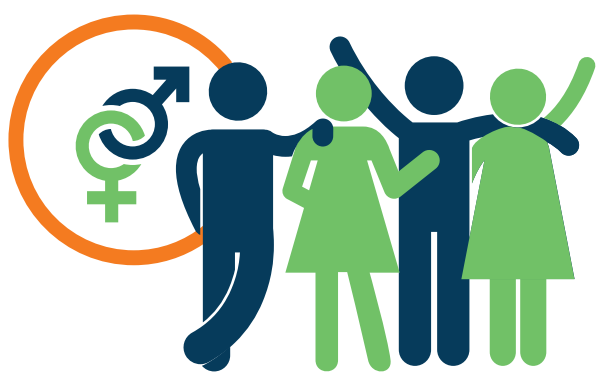

\section{Adolescent perspectives on RH and FP programs and messages: What do young people prefer?}

We found that OSY obtain information from a multitude of sources including social media, the internet, and interpersonal communication with peers and parents. OSY have very specific preferences about how they would like to receive information about RH and FP:

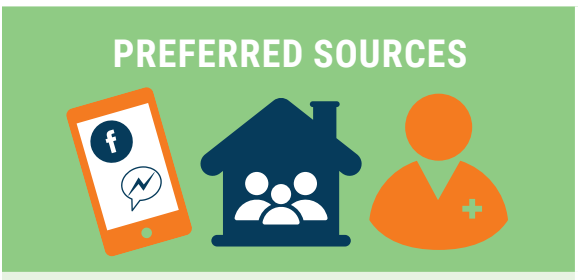

OSY prefer sources that are: easily accessible, anonymous, trustworthy, and relatable.

- Social media platforms (e.g., Facebook) and messaging apps (e.g. Messenger) provide easy accessibility and anonymity.

- OSY also seek information from interpersonal sources whom they consider relatable and trustworthy (parents, peers) or to have credibility (healthcare workers).

\section{MESSAGE FRAMING}



OSY prefer call-to-action messages framed as questions that provide information on how to get further information, services, or support.

- OSY prefer messages that are lossframed messages about unfavorable consequences of risky behaviors.

- OSY report that visual messages of negative consequences and how to avoid or mitigate these consequences will have the most impact on changing behaviors.

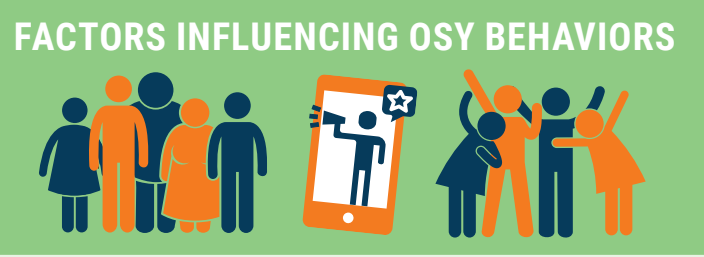

Social interactions with credible and caring peers and family members and information from social influencers impact the behaviors of $0 S Y$.

- Peers may have positive or negative influences on OSY behaviors. Peer influences are negative when substance abuse or alcohol consumption is involved.

- Despite growing influence of social media, OSY continue to consume traditional forms of media, e.g., television, movies, and radio, as well.

- Media and entertainment personalities are key influencers of OSY's RH and FP behaviors. 


\section{Social media and youth risk behaviors}

\section{OSY prefer, and enjoy access to, social media for information regarding AYRH. However, there are some down- sides to social media as well:}

- Information on reproductive health and family planning behaviors on social media may be inadequate, misleading, or inaccurate.

- Social media is one of the primary mediums where young people initiate risky behaviors (e.g., on Facebook and in messaging groups). Some young people exchange explicit images, videos, and messages through social media and communicate with each other on social media regarding these risky behaviors.

\section{How can this information be used to address the RH and FP needs of OSY in the Philippines?}

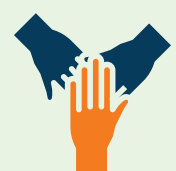

Work together: Existing programs reaching OSY with life skills and basic education should partner with programs tailored to the $\mathrm{RH}$ and FP needs of OSY, especially for those aged 15 to 19.



Get the framing right: Social media messages should be be framed according to the preferences of young people, for example, using call-to-action messages that are framed as questions and loss-framed messages about unfavorable consequences of risky behaviors. Integrate messages about substance abuse where relevant.

$2 \Omega \Omega$ Expand your audiences: AYRH programs 2838 should also target those who OSY report to be trusted advisors, including parents, peers, and healthcare workers with messages on how to speak to youth about these issues.

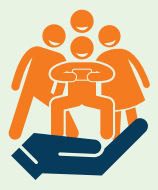

Keep youth protected: Programs should monitor and, if possible, work directly with popular social media platforms such as Facebook, to ensure that they are safe, do not encourage risky behaviors, and protect young people from inaccurate and misleading information.

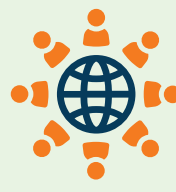

Respect the culture: SBC programming must be designed to be sensitive to the cultural, social, and religious norms of the Philippines, recognizing that culture is a central theme in how reproductive health and FP behaviors are shaped.

Evaluate effectiveness: It is critical that these programs are evaluated for effectiveness in order to ensure that only programs that have proven success are scaled up and expanded, and programs with limited success are refined and improved.



Breakthrough RESEARCH is made possible by the generous support of the American people through the United States Agency for International Development (USAID) under the terms of cooperative agreement no AID-0AA-A-17-00018. The contents of this document are the sole responsibility of Breakthrough RESEARCH and Population Council and do not necessarily reflect the views of USAID or the United States Government.
POPULATION COUNCIL

Ideas. Evidence. Impact.

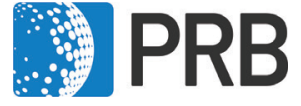

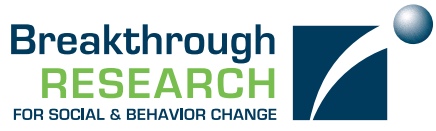

Breakthrough RESEARCH catalyzes social and behavior change (SBC) by conducting state-of-the-art research and evaluation and promoting evidence-based solutions to improve health and development programs around the world. Breakthrough RESEARCH is a consortium led by the Population Council in partnership with Avenir Health, ideas 42, Institute for Reproductive Health at Georgetown University, Population Reference Bureau, and Tulane University.

\section{Breakthrough RESEARCH Population Council}

4301 Connecticut Ave., NW, Suite 280 Washington, DC 20008

+1 2022379400

breakthroughactionandresearch.org 